\title{
Le Haut-Rhône français Géographie historique et gestion d'un fleuve
}

\author{
J.P. Bravard Université Jean Moulin. Lyon III
}

Cette étude de géographie historique concerne le Rhône entre Genève et Lyon. Les relations de l'homme avec l'environnement fluvial et la part des facteurs humains dans la dynamique du changement fluvial sont examinées. Ce travail, dont le présent article est le résumé, a obtenu le $2^{e}$ prix Henri Milon de la Société hydrotechnique de France pour 1987 (ex-aequo avec l'étude de Babacar Dieng, dont le résumé est publié dans le présent numéro).

Conçue à l'origine comme une monographie, cette étude a souhaité combler une lacune dans la connaissance des grands cours d'eau français; ce tronçon du Rhône s'étend de Genève à Lyon, sur une distance de $192 \mathrm{~km}$ au contact de " pays " ou provinces dont la spécificité a longtemps occulté la profonde originalité du fleuve. Le thème général de l'étude est l'ensemble des relations que l'homme a établies avec l'environnement fluvial depuis l'époque moderne: relations difficiles et défiantes des sociétés traditionnelles dotées de faibles moyens techniques et donc d'une faible emprise sur le milieu; relations conquérantes et dominatrices de l'ère technicienne inaugurées dès la fin du $18^{e}$ siècle et amplifiées dans la deuxième moitié du $19^{\mathrm{e}}$ siècle. Cette étude de géographie historique a tenté de déterminer la part des facteurs naturels et humains dans la dynamique du changement fluvial. L'objectif serait atteint si la prise en compte des évolutions dans la longue durée devenait une composante utile des études d'aménagement fluvial.
Après une rapide présentation du contenu de l'ouvrage, on proposera quelques réflexions sur la notion d'impact en milieu fluvial et sur la gestion des milieux naturels dans ce type d'environnement.

1. Un grand cours d'eau du piedmont alpin valorisé depuis des siècles

Le Haut-Rhône français est très représentatif de cet ensemble de cours d'eau qui drainent l'avant-pays de la chaîne alpine. De Genève à Seyssel, le Rhône a incisé une gorge étroite et profonde dans la moraine wûrmienne et son substrat molassique et calcaire. A l'aval de Seyssel, les glaciations quaternaires ont creusé une série d'ombilics à la faveur de dispositions structurales favorables: dès le Post-Glaciaire, les cours d'eau affluents, et en particulier l'Arve et le Fier, ont fourni une

Cet article reprend les thèmes principaux d'un doctorat d'Etat intitulé : « le Haut-Rhône français : dynamique naturelle et impacts des travaux d'aménagement d'un fleuve et de sa vallée " (Université Lyon III, 1985), publié sous une forme réduite en 1987, sous le titre: Le Rhône, du Léman à Lyon (Editions la Manufacture, 13, rue de la Bombarde, 69005 Lyon), 450 p.

Bravard (J.P.). - The French Haut-Rhône : historical geography and management of a river.

This historical geographical study concerns the River Rhône between Geneva and Lyon, human relations with the fluvial environment and that part of human factors as regards the dynamics of fluvial change are studied. This study, this article merely being a summary, was awarded the Henri Milon's second prize in 1987 from the Société hydrotechnique de France (ex-aequo with the study of Babacar Dieng whose summary is published in this issue). 


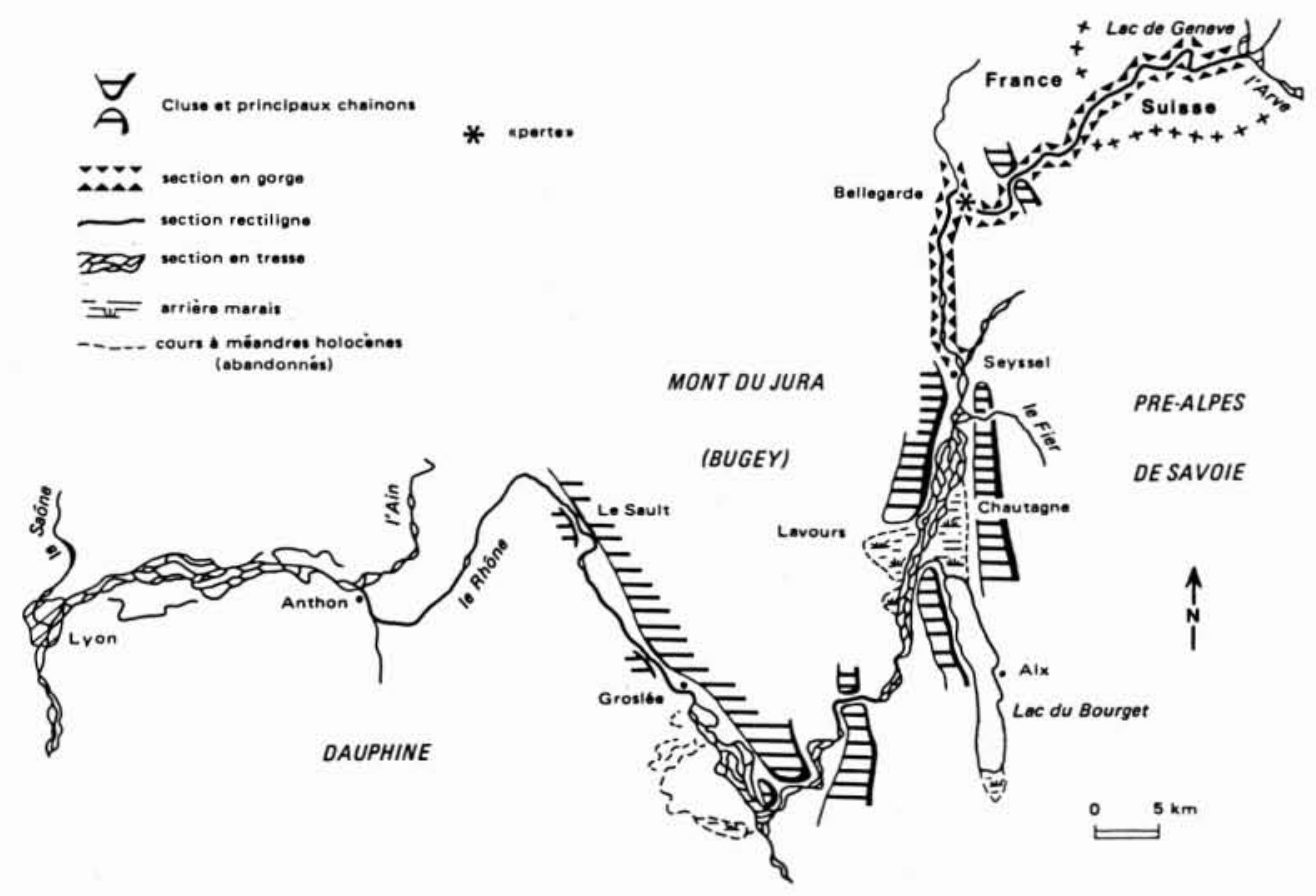

1. Les styles fluviaux du Haut-Rhône au $18^{\circ}$ siècle.

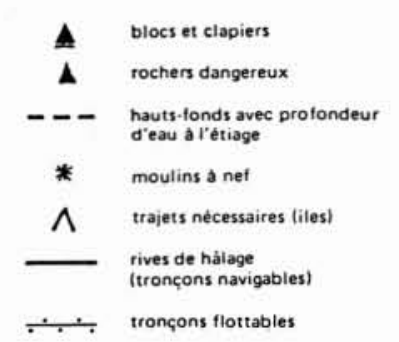

1. R. de Milliet

2 - Molard de laGreneuve

3- A la Filoche

4. R. de la Patanna
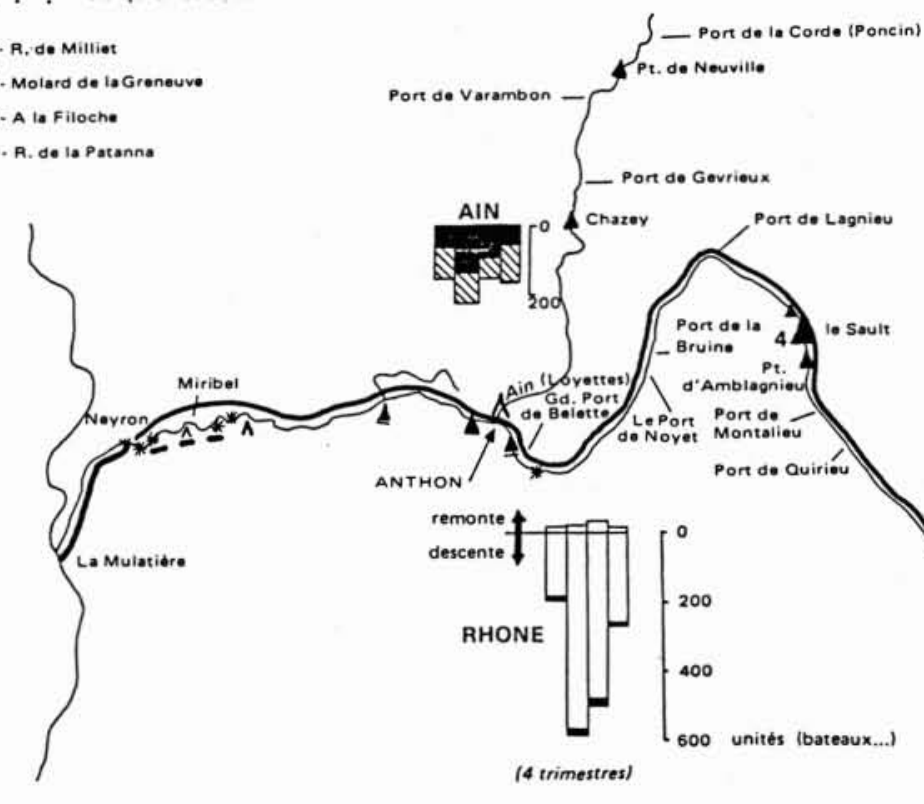
Mouvements des bateaux, barques et
radeaux chargés recensés a Anthon

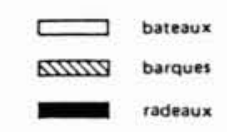


abondante charge grossière qui a progressivement remblayé les ombilics, exhaussé le lit majeur et provoqué l'isolement des vastes arrière-marais de Chautagne et Lavours. A l'aval du Sault, en revanche, le fleuve a incisé son lit dans un complexe de terrasses fluvioglaciaires car la rétention de sa charge de fond dans les ombilics amont lui a toujours conféré une énergie renforcée.

L'hétérogénéité géomorphologique de la vallée du Haut-Rhône ne doit pas occulter le caractère unificateur qui est la torrentialité du cours d'eau. A l'exception des tronçons contraints par le cadre structural, $80 \%$ du cours du Rhône développait un style tressé, ce de manière remarquable lorsque la pente du plancher alluvial excède 0,001 . La pente est le facteur sans doute le plus important mais la nature caillouteuse du fond et de la charge, comme les caractères du régime hydrologique étaient des paramètres favorables. M. Pardé a démontré qu'au début de ce siècle le Rhône conservait les caractères d'un puissant cours d'eau montagnard le module est de $450 \mathrm{~m}^{3} / \mathrm{s}$ à Lyon - doté d'une forte abondance spécifique entretenue par les affluents alpestres et jurassiens; le régime glaciaire et nival à hautes eaux de saison chaude est progressivement tempéré par des affluents aux crues de saison froide. Le Léman retarde les hautes eaux de fusion glaciaire, modère le Rhône valaisan en écrêtant et en étalant les crues, action bénéfique que le lac du Bourget et les plaines d'inondation prolongent à l'aval. Au $18^{\mathrm{e}}$ siècle, le Rhône était un fleuve peu hospitalier car de fortes contraintes naturelles retardaient les efforts séculaires de valorisation du lit majeur. L'observation des cartes anciennes et l'étude des textes ont permis de décrire un tracé changeant, des chenaux instables qui érodaient les terres et abandonnaient des bancs de galets infertiles colonisés par les "vorgines" (PARDÊ (M.), 1925. Le régime du Rhône, étude hydrologique. Lyon. 2 vol. Inst. Et. Rhod.).

Dans le domaine agricole tout était soumission aux contraintes multiples qu'imposait la présence de l'eau, dans les terroirs de prédation qu'étaient les brotteaux et les marais, comme dans les terroirs de conquête agricole. Soumission complète dans les brotteaux où des stades de végétation pionniers et juvéniles assuraient une forte productivité en bois de chauffage et permettaient une certaine élasticité des ressources à la communauté villageoise; espaces précaires, instables qui défiaient revendications et appropriations. Soumission acceptée et même revendiquée dans les grands marais où l'eau et si possible le flux minéral en provenance du cours d'eau, garantissaient un ordre stable au profit des coteaux viticoles; non pas routine mais symbiose séculaire entre la civilisation des pentes et celle des marais. Pas exactement soumission passive mais plutôt acceptation du risque dans les terroirs de conquête où le paysannat en mal de céréales cherchait à imposer dans le lit majeur le modèle rural des terres céréalières; acceptation et combat constant pour repousser le fleuve à défaut de contrôler ses débordements. Ces relations entre les riverains et leur environnement n'étaient pas univoques malgré l'apparence d'un fort déterminisme car cette société modifiait les équilibres naturels de manière sensible. Malgré la faiblesse des moyens d'intervention l'agriculture exerçait une action en retour sur les milieux humides: la destruction systématique des forêts allu- viales à bois dur diminuait le stock d'espèces spécifiques et appauvrissait la composition floristique, favorisait les stades pionniers et juvéniles, en particulier les saulaies. Dans les marais, la mosaïque des phytocénoses et la richesse de l'avifaune tenaient à cette pression discrète mais efficace.

Au $19^{\mathrm{e}}$ siècle, le Rhône et ses affluents semblent avoir possédé des populations de poissons plus proches de l'état naturel que de nos jours mais déjà fortement perturbées par une longue et intense pression humaine. Les excès de la pêche réglementée, le braconnage et les destructions chimiques dans les bras peu profonds du Rhône et de l'Ain, le rouissage du chanvre et du lin en eau calme ne pouvaient manquer d'affecter les peuplements.

Dans le domaine énergétique, les eaux du Rhône étaient sous-utilisées car les Ponts et Chaussées avaient arbitré en faveur de la navigation, fonction incompatible avec la précédente. Seules les annexes fluviales, lônes et ruisseaux affluents contribuaient véritablement à produire la force motrice nécessaire aux villages et aux bourgs car les moulins à nef étaient d'un faible secours.

La fonction essentielle du Rhône était donc la navigation. Le trafic s'opérait pour l'essentiel en "décize ": matériaux de construction et bois de chauffage pour Lyon. Très peu de marchandises remontaient le fleuve aux eaux rapides, inconstantes et semées d'embûches comme les rapides du Sault. En fait, Lyon avait fort peu de choses à proposer et imposait à l'amont une forme d'économie de traite.

Le Rhône et sa plaine alluviale étaient donc valorisés par l'homme mais demeuraient très peu aménagés au début du $19^{\mathrm{e}}$ siècle. La protection des terres agricoles n'était qu'esquissée en Chautagne depuis les années 1780; les quelques ouvrages réalisés concernaient le franchissement du fleuve et la protection de Lyon, amorcée sur la rive gauche dans les années 1830 et perfectionnée après les dégâts occasionnés par la crue centenale de 1856 . La loi de 1858 devait d'ailleurs, en assurant le maintien d'un vaste champ d'inondation naturel à l'amont de l'agglomération, interdire durablement l'aménagement contre les crues.

En fait l'essentiel des efforts du $19^{\text {e }}$ siècle a concerné l'amélioration de la navigabilité. Au $18^{e}$ siècle, le Haut-Rhône avait fait l'objet des mêmes sollicitations que les autres fleuves du territoire national. Le cul-de-sac intra-montagnard devait s'ouvrir au commerce de transit entre la Suisse et la Méditerranée; à cette fin, la section du Rhône flottable entre le Léman et le Parc fut explorée et étudiée par des savants, des ingénieurs militaires et civils, par des négociants mais la difficulté exceptionnelle des lieux et la barrière frontalière plus ou moins entrebaillée suivant l'époque, ont fait obstacle à tout investissement.

L'effort national s'est porté sur le tronçon navigable du Haut-Rhône, plus précisément sur la section Lyon-Chanaz prolongée par le canal de Savières et le lac du Bourget, vers Aix-les-Bains, porte de la Savoie. De 1848 à 1890, l'Administration des Ponts et Chaussées, malgré le manque de moyens financiers, a progressivement corrigé les deux handicaps du Rhône qu'étaient les rapides du Sault et le manque de tirant d'eau à la traversée des plaines de tressage. Euvre curieuse 


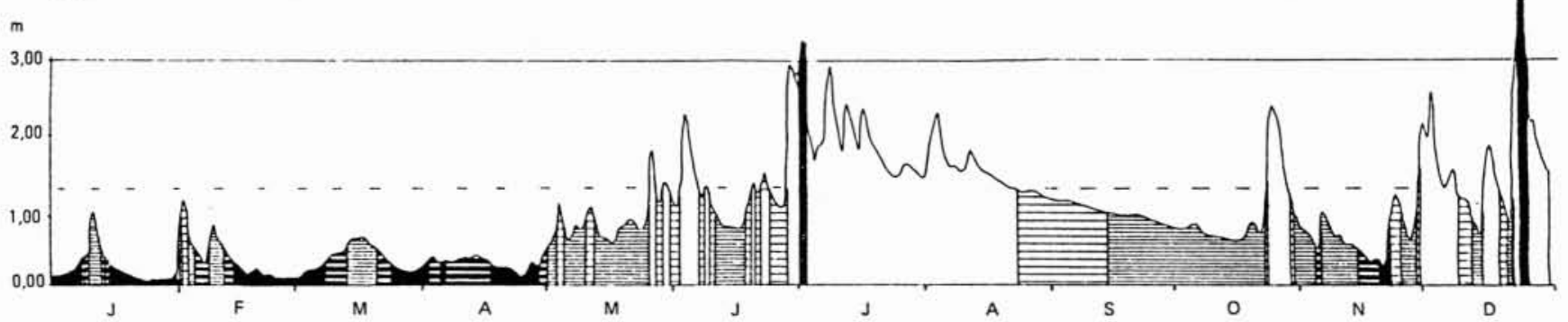

1. interruption par detaut d'eau

2. navigation difficile et dangereuse 政 Chemin de For

3. navigation passable avec

the 23 de charpement

4. nevigation assez bonne.

chergement encore incomplet

5. navigation complate.

6. interruption per les cruen

1851 à 1858

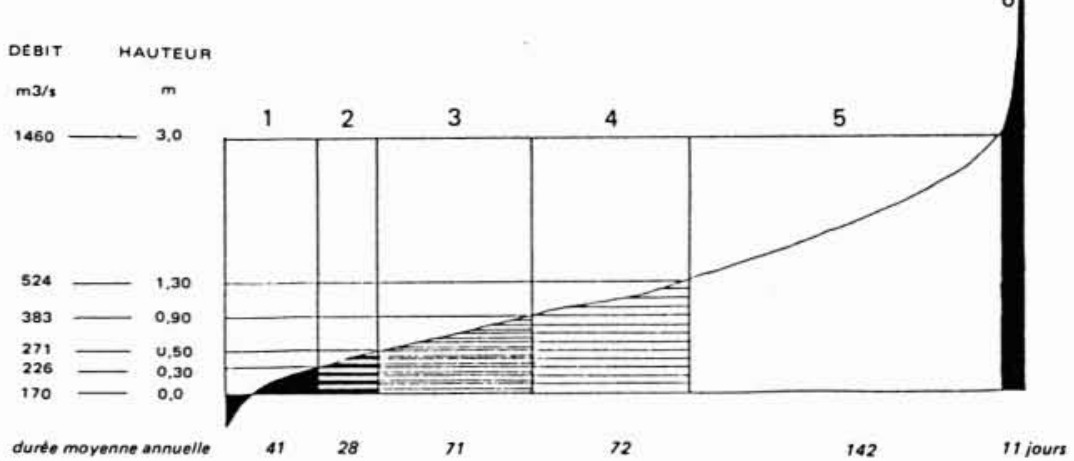

3. a) Relation entre la cote du Rhône au pont Morand (Lyon) et la navigabilité en 1854. L'hydrogramme est représentatif du régime nivoglaciaire avec des hautes eaux de saison chaude bien marquées et un étiage hivernal qui interdit quasiment la navigation de Janvier à Avril; noter l'intérêt des crues automnales pour les mariniers, malgré le danger qu'elles représentent.

b) Durée moyenne annuelle des débits navigables à Lyon (période 1851-1858)

(Source : service de la Navigation du Rhône et de la Saône).

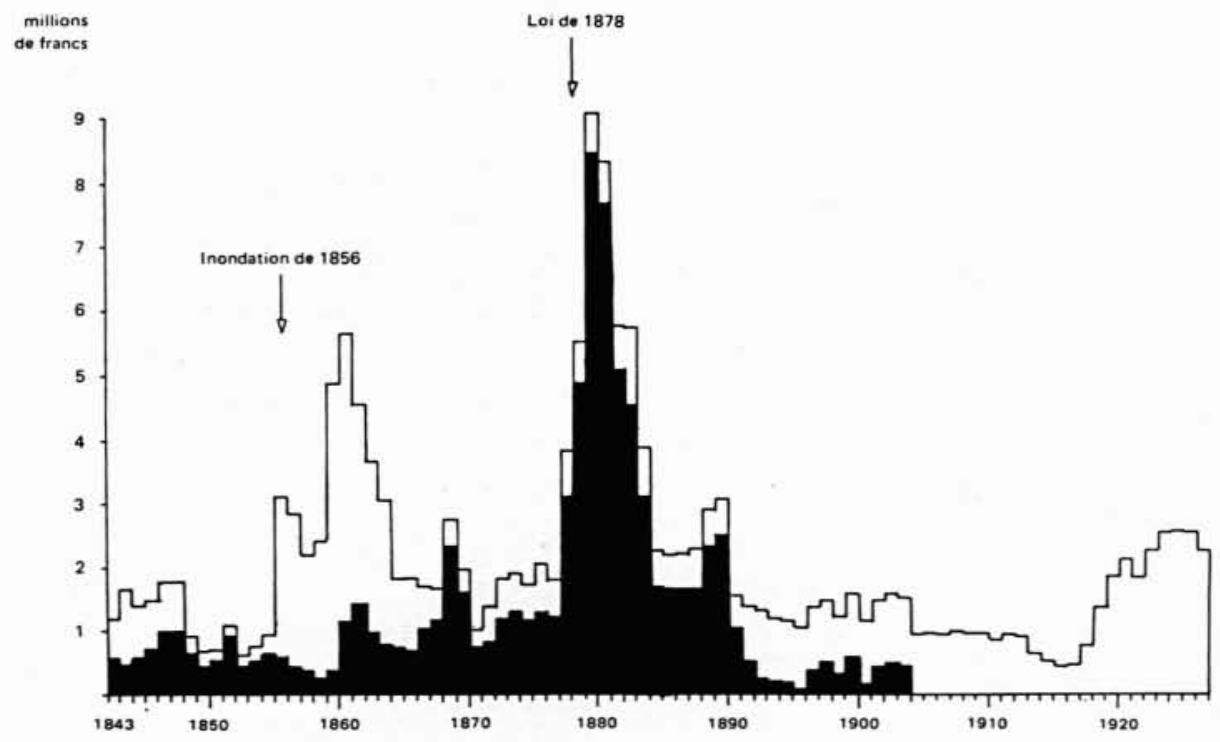

4. Dépenses effectuées par les Ponts et Chaussées sur le Haut-Rhône entre 1843 et 1926 . Les travaux de correction fluviale ont cessé en 1904. 


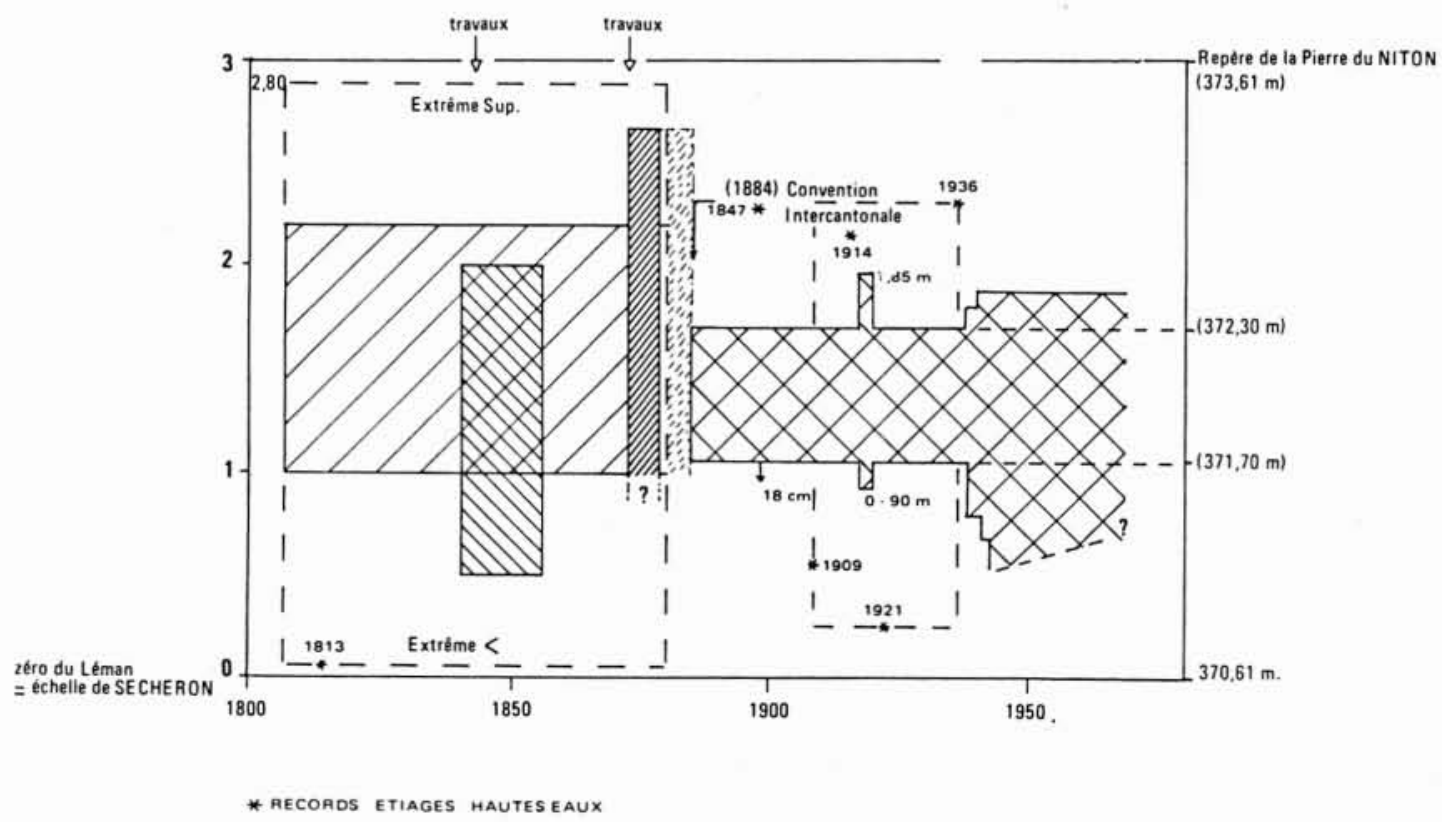

5. Les réglementations successives concernant les niveaux du lac Léman à Genève depuis le début du $19^{e}$ siècle. Les blocs hâchurés représentent la marge et les niveaux de fluctuation moyens. La convention intercantonnale de 1884 a réduit la capacité de stockage du lac mais une évolution inverse est apparue à la fin des années 1930; ceci permet un écrêtage des débits d'été et un meilleur soutien aux étiages de fin d'hiver. marquée par les efforts redoublés en faveur d'une voie d'eau condamnée dès la fin des années 1850 par l'essor du trafic ferroviaire; investissements détournés du Rhône aval au profit d'un trafic moribond... Le HautRhône a coûté fort cher pour un simple trafic de pierre de taille et de chaux.

A la fin du $19^{\circ}$ siècle, les Suisses ont amélioré leur contrôle sur les niveaux du lac Léman de manière à augmenter la production hydroélectrique de l'émissaire genevois. Dans ce domaine, le Rhône peut être considéré comme l'un des berceaux de la houille blanche. Le secteur le plus attractif était les gorges du tronçon amont, équipées d'un barrage dès 1871 , puis sujettes à une active compétition entre investisseurs genevois, lyonnais et parisiens. Alors que le tronçon suisse et franco-suisse s'équipait de barrages, le tronçon français demeuré sous-équipé au début du $20^{\mathrm{e}}$ siècle et la valorisation de l'énergie produite à Bellegarde n'intéressait que la production électrochimique.

A l'aval, les projets ont abondé mais la seule œuvre hydraulique réalisée fut l'aménagement de Jonage-Cusset (1899) destiné à satisfaire la demande lyonnaise en électricité.

Ainsi le caractère le plus remarquable du Haut-Rhône est bien ce sous-équipement durable du potentiel hydraulique qui se prolongea jusqu'à la réalisation de Génissiat. Le fleuve fut véritablement mis en réserve pendant de longues décennies; les Ponts et Chaussées voulurent ménager le plus longtemps possible les intérêts de la navigation qu'ils avaient mission de défendre et de promouvoir; ils voulurent également décourager les initiatives hasardeuses et spéculatives car leur mission était de tirer le meilleur parti possible du potentiel rhodanien. Cette attitude prudente, soucieuse de l'intérêt général, a peut-être freiné le dynamisme rhodanien à l'époque où les vallées alpines prenaient leur essor. Il fallut attendre le lendemain de la guerre de 1914-1918 pour que la loi du Rhône réservât le fleuve à une Compagnie Nationale, la C.N.R., qui achève actuellement les travaux d'équipement, en complétant l'escalier de barrages réalisé à l'aval de Lyon.

Une autre singularité du Haut-Rhône, mais elle a pu se manifester dans d'autres vallées alluviales, est un déclin relatif survenu entre la fin du $19^{\mathrm{e}}$ siècle et les années 1950. En effet, la phase de récession de la navigation et de freinage de l'équipement hydroélectrique a coïncidé avec une sévère crise des campagnes, phénomène national, qui s'est traduite dans les plaines du Haut-Rhône par une modification des relations que l'homme entretenait avec les milieux humides. Par un renversement de situation unique depuis des siècles les terres proches du fleuve n'étaient plus un espace de conquête à partir des années 1860-1880; les labours se mirent à reculer devant les herbages. Les causes sont multiples: tassement démographique, fin de l'autarcie alimentaire, crises de la vigne puis saignée démographique de la guerre. Cette perte de dynamisme local se conjuguait avec le refus de l'Administration de lever la contrainte de l'inondation. La tendance s'est à nouveau renversée depuis une trentaine d'années: un meilleur contrôle des nappes phréatiques, le recours aux fertilisants artificiels, la mécanisation ont revalorisé les terres humides qui se sont spécialisées dans la culture du maïs et la populiculture. Par un effet de convergence remarquable la société contemporaine retourne au fleuve; l'équipement hydroélectrique, le refroidissement des centrales nucléaires, l'exploitation de l'eau potable et des ressources en granulat dans les périphéries urbaines, l'essor des loisirs aquatiques matérialisent cette évolution profonde. 


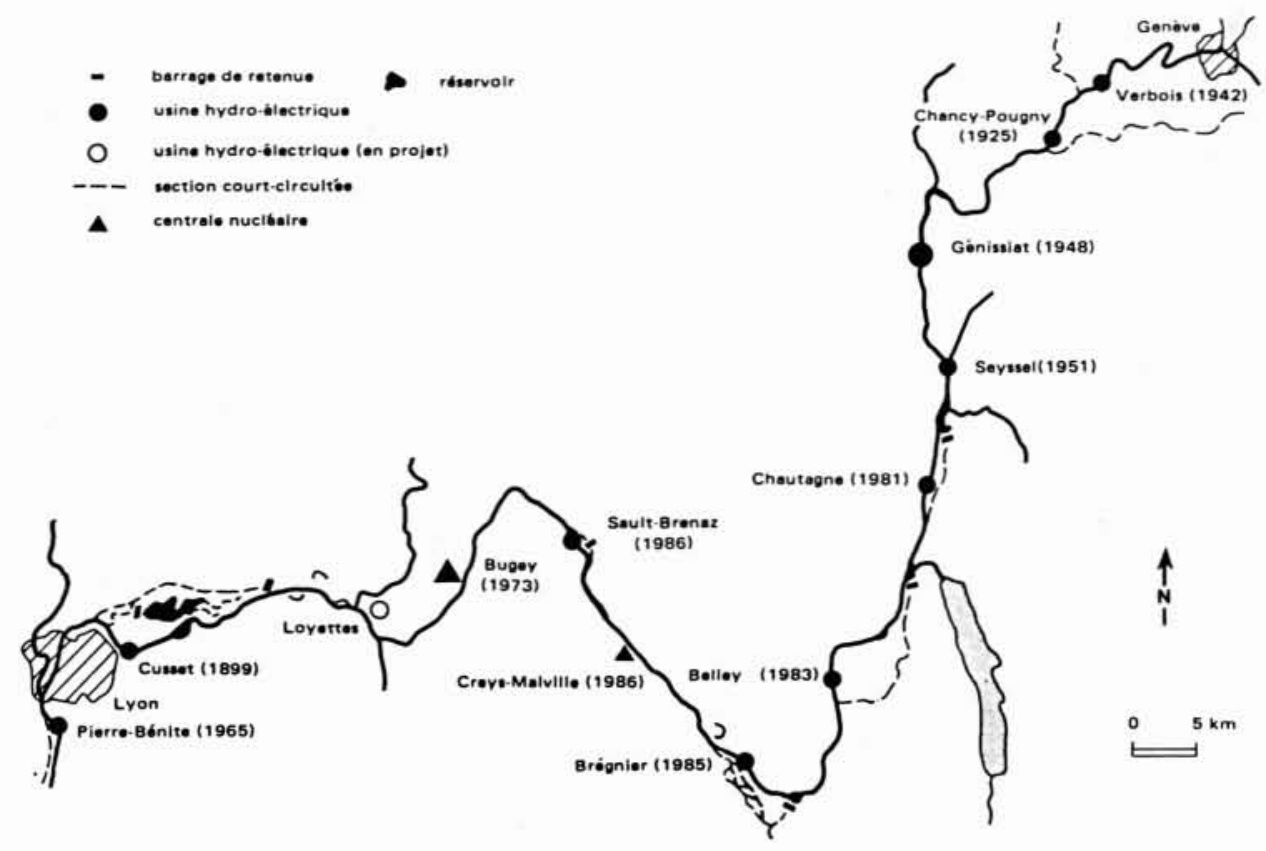

6. Les aménagements réalisés au $20^{e}$ siècle sur le Haut-Rhône.

\section{Les impacts des interventions humaines}

Les travaux de génie civil réalisés de manière localisée du $18^{\mathrm{e}}$ siècle à la fin du $19^{\mathrm{e}}$ et la construction des réservoirs engagée au $20^{\mathrm{e}}$ siècle ont perturbé le transit de la charge de fond caillouteuse et réduit son volume à quelques dizaines de milliers de $\mathrm{m}$ sur les tronçons où le lit est mobile à l'aval de Génissiat. L'exemple le plus notoire est le basculement du canal de Miribel (1848-1858) mais ce processus a touché, de manière moins sévère, la Chautagne et une grande partie du cours jurassien.

Cette question a perdu récemment une partie de son intérêt car l'achèvement de l'escalier de barrages hydroélectriques a stabilisé les fonds; en revanche se pose depuis une quarantaine d'années la question difficile du transit des sédiments en suspension (en moyenne 400000 T/an. La décantation dans les bassins de Verbois et Génissiat est en premier lieu sélective, dans la mesure où le transit ne concerne plus que les classes de tailles inférieures ou égales aux sables moyens; les conséquences écologiques induites par une sédimentation alluviale plus fine sont réelles mais restent secondaires si on les compare à l'effet des vidanges de réservoirs. La technique des chasses triennales est maitrisée sur les réservoirs amont mais la présence des retenues à dérivation impose à la Compagnie Nationale du Rhône des ajustements très délicats pour épargner les sections courtcircuitées, tout en assurant le transit de la MES le plus loin possible vers l'aval. Les contraintes imposées par les impératifs écologiques, surtout depuis la vidange de 1978, comme par la présence de centrales nucléaires refroidies par les eaux fluviales, font partie de ces questions émergeantes qui rendent nécessaire une adaptation constante des aménageurs et des défenseurs de l'environnement.
Sur le plan hydrologique, les changements concernent essentiellement le contrôle du régime par la gestion du lac Léman et par la construction des réservoirs suisses d'altitude; globalement, entre 1892 et les années 1940, une réduction de la capacité de rétention du lac Léman a augmenté les débits de saison chaude; un phénomène inverse s'est produit depuis cette époque car la capacité totale de rétention, portée de 340 à $1700 \mathrm{Mm}$, a permis un stockage des débits de fusion nivo-glaciaire et une hausse des débits de saison froide. Le Rhône a donc enregistré une régularisation sensible de son régime depuis environ un siècle; son hydrologie est également influencée par la gestion des usines hydroélectriques qui, depuis la fin des années 1880 , imposent des pointes quotidiennes de débit. La fréquence et l'intensité des épisodes exceptionnels n'a pas été sensiblement réduite car le bassin-versant français a été l'objet d'un équipement limité. Depuis les crues de 1840 et 1856 , la politique de protection s'est toujours appuyée sur une protection absolue des grands champs naturels d'expansion des crues (275 km à l'état naturel entre Genève et Lyon) et, malgré quelques entorses récentes à ce principe dues à la pression des intérêts locaux, la situation devrait avoir peu évolué; en réalité l'incision du lit mineur et certains endiguements ont réduit la fréquence et la durée de mise en eau de plusieurs plaines pour des débits d'une période de retour voisine de 1 à 10 ans.

Les biologistes enregistrent l'évolution des biocénoses des îles alluviales: l'abaissement localisé de la ligne d'eau, la réduction des submersions modifiant les conditions hydriques et la quasi-suppression du renouvellement des formes fluviales (construction des bancs alluviaux) se conjuguent pour provoquer une rapide évolution vers une forêt de bois durs homogène. Les marais drainés sont soit revendiqués par les agriculteurs, soit colonisés par des saulaies - aulnaies après l'abandon des pratiques traditionnelles. 
Les analyses physico-chimiques régulièrement pratiquées sur le Haut-Rhône reconnaissent la bonne qualité de ses eaux; le taux d'oxygène dissous est très élevé, la DB05 faible, la minéralisation est forte mais naturelle, l'état sanitaire satisfaisant. Ces caractères, joints à d'autres paramètres favorables comme la pente, la vitesse et la température de ses eaux étaient sans doute favorables, dans la succession des secteurs tressés, à des peuplements piscicoles de type "zone à Ombre " et dans les secteurs plus lénitiques à des peuplements de type "zone à Barbeau ". Les biocénoses se sont appauvries malgré ce potentiel favorable : la gestion déraisonnable de la pêche ancienne, la chenalisation des secteurs tressés, l'aménagement hydroélectrique ont cumulé leurs impacts depuis le $19^{\mathrm{e}}$ siècle; cette situation justifie la gestion écologique des sections court-circuitées et des milieux qui ont conservé un intérêt biologique.

\section{Aménagements fluviaux, changements de l'environnement et gestion des mi- lieux naturels}

Depuis la loi de juillet 1976, les aménagements d'une certaine importance nécessitent la réalisation d'une étude d'impact qui doit établir un état des lieux avant l'intervention projetée et prévoir ses effets sur l'environnement. Cet état "zéro " est le plus souvent caractérisé par la description de quelques paramètres du milieu, jugés pertinents suivant les critères du moment, les moyens financiers dont dispose le maître d'œuvre et les compétences dont il souhaite s'entourer à cette occasion. Dans le souci légitime d'évaluer l'impact futur des aménagements - c'est la pratique du "suivi écologique " calé sur l'état de référence - on a trop souvent tendance à sous-estimer le fait que la perturbation va s'insérer dans un environnement par nature instable. En réalité, depuis des siècles, les cours d'eau tempérés et leurs plaines alluviales ont subi des perturbations sensibles de telle sorte que la dynamique actuellement analysable enregistre à la fois le fonctionnement de l'écosystème et une dérive plus ou moins rapide imputable à l'impact des interventions humaines. Ainsi toute étude d'impact en milieu fluvial - pour se cantonner à ce type d'environnement - devrait situer les paramètres analysés dans le cadre d'un complexe naturel et dans une perspective historique longue. Une telle préoccupation est encore rare chez les maîtres d'œuvres, mais elle présente souvent l'avantage - non négligeable au moment des prises de décision - de relativiser les impacts contemporains. (Amoros et al., 1986; Bravard et al., 1986; Amoros et al., 1987 Pautou et al., 1987).

Considérons, à titre d'exemple, le changement subi par les sections fluviales rhodaniennes caractérisées au $18^{e}$ siècle par un style en tresse. Alors que les éperons et autres "digues paysannes " limitaient à grand peine l'érosion des berges sur les marges d'une bande active large de plusieurs centaines de mètres, voire de plusieurs kilomètres, les digues submersibles construites pour améliorer les conditions de navigabilité ont transformé le style fluvial. Ces digues ont concentré les eaux d'étiage dans le chenal de navigation et barré l'entrée amont des chenaux secondaires; il en est résulté, dans la plupart des cas, une incision du chenal principal, un remblaiement des chenaux annexes par les matières minérales en suspension et une série d'impacts indirects tels que l'abaissement de la nappe phréatique, la stabilisation complète des berges par l'arrêt des processus d'érosionsédimentation, le développement et le vieillissement de la forêt alluviale sur des îles et des berges exhaussées par les dépôts de crue. Si les liens de causalité entre l'aménagement et le changement de l'environnement sont clairement établis et bien délimités dans l'espace, il est nécessaire de considérer le cours d'eau comme un système dans lequel les sections sont interdépendantes; dans le cas présent, cette remarque signifie que les secteurs de tressage ont également enregistré l'évolution pluri-séculaire du transport de la charge de fond et de la charge en suspension ainsi que les fluctuations du régime hydrologique: les phases de défrichement et de reboisement du bassin-versant alpin, les fluctuations climatiques marquées par des avancées et des reculs glaciaires, par des variations de l'écoulement, la construction des réservoirs, ont ainsi fait varier les paramètres du tressage que sont le débit et la charge. Le paysage fluvial actuel des anciens secteurs de tressage n'est qu'un état transitoire, déterminé par le jeu de paramètres qui sont, pour partie, méconnus.

Ces remarques posent la question de la réalité objective du changement et de sa perception par la société. Les mutations sont toujours lentes, à l'échelle humaine, souvent différées dans le temps, et plus ou moins intenses. Au début du $19^{\mathrm{e}}$ siècle des travaux de génie civil d'ampleur limitée provoquaient en général les vives réactions des populations riveraines inquiètes d'une perturbation des équilibres physiques et surtout économiques; réactions sans doute abusives qui anticipaient sur la réalité et l'ampleur des changements. "A contrario " le creusement du canal de Miribel, entre 1848 et 1858 , fut un ouvrage considérable pour l'époque qui ne suscita pas d'opposition de cette nature; le changement ne fut perçu, puis analysé, que de manière très indirecte lorsque, une quinzaine d'années après l'achèvement des travaux, il fut patent que la partie aval de la plaine alluviale proche de Lyon enregistrait une sérieuse aggravation des crues; le basculement du profil en long et l'exhaussement du lit majeur avaient élevé le niveau des eaux à débit égal. Cet exemple démontrait que le changement peut être révélé incidemment après la prise de conscience d'une mutation révélatrice. La remarquable précision des mesures effectuées à l'occasion des travaux permit à l'ingénieur $\mathrm{H}$. Girardon d'effectuer la démonstration de ce basculement car il disposait de l'état des lieux pouvant servir de référence, mais combien d'impacts ne sont pas analysés faute d'éléments de comparaison. Ceci pour suggérer que la non-perception du changement est trop souvent la règle, soit que la mutation soit trop lente ou différée, soit que la démonstration, qui nécessite un investissement à moyen et long terme, ne paraisse pas pertinente aux décideurs et aux agents économiques. La situation est d'une acuité particulière dans le domaine biologique car les moyens 
d'investigation sont délicats à mettre en œuvre en milieu fluvial, et car les progrès scientifiques sont trop récents pour que des comparaisons puissent être réalisées sur le temps long.

Enfin une perspective historique présente de l'intérêt dans le débat récent qui porte sur l'entretien des écosystèmes fluviaux et sur une éventuelle restauration. En théorie, la conservation et la restauration supposent que soient clairement définis des états de référence, si possible proches des conditions naturelles pour la seconde. Les tentatives se multiplient en Europe pour régénérer certaines sections de tressage profondément transformées par les endiguements du $19^{e}$ siècle; la méthode suggérée ou déjà employée est l'ouverture contrôlée des enrochements qui barrent l'entrée amont des bras secondaires. L'écoulement est ainsi réactivé par une augmentation du débit et des vitesses; cette solution prudente a le mérite d'entretenir les écosystèmes mais n'est pas une restauration qui signifierait érosion des berges, transit de charge de fond, sédimentation et création de milieux neufs sur le plan de la géomorpho- logie et des phytocénoses. C'est à ce prix que l'on pourrait approcher les conditions caractérisant le fonctionnement sinon naturel, du moins primitif. En réalité, les matériaux sont prélevés dans le stock sédimentaire du lit majeur car les apports amont ont quasiment cessé, si on compare la charge à ce qu'elle était voici deux siècles (exception faite des classes de taille les plus fines de la charge en suspension). Ce genre d'opération nécessite un contrôle hydraulique délicat mais présente un intérêt indéniable pour le simple maintien des paysages et des écosystèmes fluviaux à un stade d'évolution relativement avancé; un certain rajeunissement est possible mais ne permet pas une restauration, au sens propre du terme. Ce bref exemple pose une nouvelle fois la question de l'état " naturel " que les fleuves des pays industriels ont définitivement perdu. La réversibilité, c'est-à-dire, dans ce cas, le retour à des conditions primitives, serait à la rigueur possible sur les têtes de bassin mais n'est pas réaliste sur les rivières aménagées; la notion même de "tiers sauvage " ou la politique des réserves naturelles doivent tenir compte de ces réalités.

\section{Bibliographie}

Amoros C., Reygrobellet J.L., Bravard J.P., Pautou G., Roux A.L., 1986: Methodological research applied to ecological management of water resources of fluvial systems. Regulated rivers $\mathrm{I}, 17-36$.

Bravard J.P., Roux A.L., Amoros C., Coulet M., Reygrobellet J.L., Bournaud M., Pautou G., 1986 : Evolution spatio-temporelle des systèmes fluviaux aménagés : recherches méthodologiques sur le Haut-Rhône français. La Houille Blanche, XIXe Journées de l'hydraulique, IV, 18, 8 p.

Amoros C., Rostand J.C., Pautou G., Bravard J.P., 1987 : the reversible process concept applied to the environmental management of large river systems. Environmental management. (sous presse).

Pautou G., Roux A.L., Amoros C., Bravard J.P., Coulet M., 1987 : Vers une gestion écologique des plaines alluviales du Haut-Rhône français : les apports de la recherche. Colloque Conseil de l'Europe - WWF, Rastatt, 17-20 sept. 12 p. (soumis pour publication).

Adresse de l'auteur :

Monsieur J.P. Bravard

Université Jean-Moulin, Lyon III

Faculté des lettres et civilisations

74, rue Pasteur - BP 0638

69239 Lyon Cedex 02

L'illustration a été réalisée par le service de dessin du LA 260 "géographie rhoda. nienne " du C.N.R.S. 